\title{
Movimentos Oculares no Bebê: o Que Eles nos Indicam Sobre o Status Oftalmológico e NeurolóGico
}

\author{
Marcelo Fernandes Costa ${ }^{1}$ \\ Instituto de Psicologia - USP
}

\begin{abstract}
Movimentos oculares anormais podem ser o resultado de um desenvolvimento visual anormal ou estar sinalizando a existência de uma doença neurológica ou neuromuscular. Assim, é importante que o clínico conheça essas anormalidades e possa distingui-las dos movimentos oculares normais, porém imaturos. Este trabalho oferece esclarecimentos sobre os diferentes tipos de movimentos oculares, como interpretá-los nos bebês saudáveis e quais os tipos de alterações encontradas nas diferentes doenças visuais e neurológicas.
\end{abstract}

Descritores: Movimentos oculares. Bebês. Distúrbios da visão.

\section{Introdução}

Sistema oculomotor no neonato saudável é imaturo. No entanto, mesmo logo após o nascimento, já é possível distinguir movimentos ainda imaturos de movimentos patológicos. A análise dos movimentos oculares, além de nos alertar sobre a presença de alguma alteração visual ou neurológica, também pode auxiliar o clínico a classificar diferentes doenças neurológicas, como as ataxias espinocerebelares - se são do tipo 1, 2 ou 3 (Harris, Taylor, \& Velodi, 1997; Rivaud-Pechoux, Durr, \& Gaymard, 1998).

O sistema oculomotor recebe entradas neurais de múltiplos sistemas:

1 Docente do Departamento de Psicologia Experimental e Núcleo de Neurociências e Comportamento do Instituto de Psicologia - USP. Endereço eletrônico: costamf@usp.br 
sistemas de sacadas, sistema de perseguição lenta, sistema vergencial, sistema optocinético e sistema vestibular. Todos esses sistemas são imaturos ao nascimento (Archer, Sondhi, \& Helveston, 1989; Cordero, Clark, \& Urrutia, 1983; Harris, Jacobs, \& Taylor, 1994; Harris, Jacobs, Walker, \& Shawkat, 1993; Von Hofsten \& Rosander, 1997).

Clinicamente, é importante avaliar todos os sistemas, mesmo que para obter um mínimo de informação sobre cada um deles. Somente uma análise completa poderá auxiliar na localização de uma lesão ou na identificação de um padrão específico de movimento ocular que caracteriza um defeito visual (Lennerstrand, Zee, \& Keller, 1982).

A importância de se diagnosticar um defeito de movimentação ocular precocemente é evitar que decorram dessa alteração prejuízos visuais funcionais. Uma vez que o sistema visual depende de estimulação para se desenvolver corretamente, alterações oculomotoras no início da vida podem acarretar em danos visuais futuramente irreparáveis (Koh et al., 1994; Lennerstrand, Zee, \& Keller, 1982).

Este trabalho está dividido em duas partes: uma inicial, em que faremos uma revisão dos movimentos oculares no bebê saudável associados a cada um desses sistemas, e uma segunda, em que trataremos de como as diversas patologias oftalmológicas e neurológicas alteram a resposta de cada sistema em particular. $\mathrm{O}$ que vamos apresentar não é um roteiro para diagnóstico ou tratamento dos distúrbios oculomotores, nem temos tal intenção. Com efeito, acreditamos ser importante aos profissionais que trabalham com crianças e bebês ter o conhecimento de que alterações nos movimentos oculares podem sinalizar uma patologia neurológica ou oftalmológica.

\section{Movimentos oculares no bebê saudável}

\section{Movimentos em sacadas}

A sacada é um movimento ocular rápido que pode ocorrer involuntariamente (sacada reflexa) ou voluntariamente (sacada voluntária), com o propósito de redirecionar a fóvea a um alvo específico. As sacadas voluntárias 
podem ser divididas em quatro classes: 1) preditiva - ocorre em antecipação a um alvo que vai aparecer em um local específico; 2) iniciada-por-comando - por exemplo, quando alguém pede a você que olhe para a direita; 3 ) guiada pela memória; 4) anti-sacada (Leigh \& Zee, 1991).

O sistema de sacadas não está totalmente desenvolvido até o final do primeiro ano de vida. Bebês até essa idade apresentam sacadas hipométricas (curtas e insuficientes para atingir o objeto-alvo) e podem necessitar várias sacadas secundárias até conseguirem fixar o alvo. Por volta do 7ํo mês de vida, observa-se uma importante e acentuada progressão para a normometria (100\% da distância do objeto-alvo) que se refina até que, por volta de 1 ano de idade, o movimento sacádico já está equiparado ao do adulto (Harris, Jacobs, \& Taylor, 1994).

A via para os movimentos sacádicos se origina no córtex visual (áreas oculomotoras frontais estão envolvidas nas sacadas voluntárias e o córtex parietal nas sacadas reflexas). Projeta-se então para a cápsula interna, dividindose em via dorsal e via ventral. O ramo dorsal vai para o colículo superior, e o ramo ventral, para a ponte e o mesencéfalo, os quais contêm os núcleos dos nervos oculomotores e estruturas do olhar conjugado, como a formação reticular paramediana pontina (FRPP) e o fascículo longitudinal medial (FLM) (Hikosaka, 1989; Moschovakis, Scudder, \& Highstein, 1996).

Acredita-se que o colículo superior seja um centro importante para a computação correta da amplitude e do meridiano da sacada (Fries, 1984). Além dessas vias, o sistema de sacada está sob contínuo controle adaptativo do cerebelo (Leigh \& Zee, 1991).

\section{Movimento de perseguição lenta}

A função do movimento de perseguição lenta é manter a fixação sobre um alvo em movimento; freqüientemente, tanto os olhos quanto a cabeça são necessários. Esse tipo de movimento é uma tarefa complexa, necessitando de processos de predição para transpor atrasos no tempo do sistema oculomotor e a capacidade de supressão do reflexo vestíbulo-ocular, já que, quando a cabeça se movimenta, os olhos são estimulados por esse movimento reflexo a ir para a direção oposta (Sokol, Peli, \& Moskowitz, 1991). 
A via visual genículo-estriada conduz a informação visual para o córtex extraestriado na áreas parietocciptotemporal, as quais são essenciais para o movimento de perseguição lenta (Morrow \& Shape, 1990). Os inputs do movimento de perseguição lenta, vindos do córtex, passam pela superfície lateral do ventrículo lateral, pela cápsula interna, pelo pedúnculo cerebral e chegam à ponte, alcançando o núcleo pontino dorsolateral, o núcleo vestibular e, finalmente, o núcleo oculomotor no tronco encefálico. O cerebelo também é importante para o início desse movimento (Leigh \& Zee, 1991).

Logo na primeira semana de vida, o movimento de seguir um objetoalvo que atravessa o campo visual de um lado para o outro já está presente. No entanto, não tem a mesma qualidade do adulto: depende de um alvo de tamanho relativamente grande e de uma baixa velocidade de deslocamento. O desenvolvimento do movimento para o olhar conjugado horizontal ocorre antes do movimento para o olhar conjugado vertical. Esse desenvolvimento é intenso nos primeiros 6 meses de vida, mas somente na adolescência é que estará completo (Braddick \& Atkinson, 1983).

\section{Movimentos vergenciais}

A vergência é a habilidade de mudar o ângulo entre os dois eixos visuais, permitindo que a imagem caia sobre a fóvea para manter uma visão única e a estereopsia tanto para estímulos próximos como para distantes (Braddick $\&$ Atkinson, 1983). Esse movimento ocular é conduzido pela disparidade da imagem retiniana e borramento visual do processo acomodativo. Além da convergência, a fixação para perto também inclui a acomodação e a miose pupilar (Smith, 1987).

Existem dois tipos de movimento vergencial: 1) convergência - ambos os olhos se movimentam na direção nasal; 2) divergência - ambos os olhos se movimentam na direção temporal. Nos neonatos, é muito comum observar momentos em que os olhos estão em posição divergente intercalados com momentos de paralelismo ocular (Leigh \& Zee, 1991). Somente por volta dos 3 meses de idade é que os movimentos convergentes aparecem. Essa instabilidade na manutenção da postura ocular desaparece até os 6 meses de vida, quando aproximadamente $97 \%$ dos bebês já não apresentam mais desvios oculares 
Movimentos Oculares no Bebê: o que Eles nos Indicam Sobre o Status Oftalmológico...

(Leigh \& Zee, 1991). Vale ressaltar que o mecanismo oculomotor que garantirá a percepção de apenas uma imagem, chamado de fusão, é muito dependente dos sistemas vergenciais e, portanto, não está presente até por volta dos 6 meses (Braddick \& Atkinson, 1983).

\section{Reflexo vestíbulo-ocular}

O reflexo vestíbulo-ocular se refere a movimentos conjugados compensatórios dos olhos, que são induzidos por movimentos da cabeça. Junto com o sistema optocinético, sua função é manter a imagem estável na retina durante os movimentos da cabeça que ocorrem naturalmente (Carpenter, 1988; Leigh \& Zee, 1991).

Trata-se de um movimento gerado pela estimulação dos canais semicirculares. Quando um sujeito é subitamente rodado, as paredes dos canais semicirculares se movem relativamente à endolinfa existente dentro do canal. Esse relativo movimento inclina as células capilares, as quais estão imersas em uma cúpula gelatinosa na ampola de cada canal. Os olhos tendem a assumir uma posição de olhar conjugado para o lado oposto ao estimulado (Barnes, 1993). Esse movimento ocular já está presente ao nascimento. Contudo, em bebês prematuros e em alguns bebês nascidos a termo, pode-se freqüentemente observar um movimento ocular conjugado vertical para cima. Suspeita-se que a causa desse movimento decorra da imaturidade do sistema sacádico ou das vias neurais que integram a informação vestibular com os núcleos oculomotores pontinos e mesencefálicos (Leigh \& Zee, 1991).

Esse movimento ocular é composto por duas fases. A primeira, chamada de fase lenta, refere-se ao desvio ocular tônico para o lado oposto ao da estimulação que mencionamos acima. A segunda compõe-se de movimentos sacádicos de reposicionamento ocular e, por isso, é conhecida como fase rápida. A fase rápida só pode ser identificada por volta dos 45 dias de vida (Barnes, 1993).

\section{Nistagmo optocinético}

O nistagmo optocinético é um movimento reflexo, fisiológico, caracterizado por um nistagmo conjugado dos olhos. Compõe-se de uma fase lenta 
e de uma fase rápida, e é gerado por estímulos visuais em movimento. A fase lenta representa o movimento de perseguição lenta do alvo até o limite do campo visual, e a fase rápida, o movimento sacádico de reposicionamento ocular. Esse movimento é naturalmente iniciado quando, por exemplo, olhamos através da janela de um automóvel em movimento (Atkinson, 1989).

O nistagmo optocinético apresenta duas vias neurais distintas, porém paralelas. Uma delas, que recebe o nome de atrasada (ou indireta, lenta), ocorre lentamente (pode demorar dezenas de segundos) e gera o nistagmo que sucede ao nistagmo optocinético, uma diminuição gradual do nistagmo que ocorre sob condições escotópicas (baixa luminosidade). Este nistagmo é muito relacionado como reflexo vestíbulo-ocular; iniciam-no inputs visuais de movimento no córtex visual que chegam via núcleo do trato óptico, no pré-tecto, e pelo núcleo vestibular. A outra via recebe o nome de inicial (ou direta, rápida), ocorrendo rapidamente (em menos de 1 segundo) e não gera o nistagmo que ocorre após o nistagmo optocinético. Aumenta muito em condições escotópicas. A sua via neural é similar à do movimento de perseguição lenta (Atkinson, 1989; Leigh \& Zee, 1991).

Nos bebês neonatos, esse movimento já pode ser observado; porém, é evidente a presença de uma assimetria dependendo da direção em que se movimenta o alvo. Nesses bebês, o nistagmo optocinético é facilmente observado se o alvo caminha na direção temporo-nasal. Somente após os 3 meses é que essa assimetria se torna progressivamente menos evidente; aos 6 meses, já não existe mais (Braddick \& Atkinson, 1983).

\section{Movimentos oculares anormais em bebês}

\section{Movimentos sacádicos}

Um fenômeno comum em sujeitos com movimentos sacádicos normais e anormais é a presença de algum grau de dismetria (perda de precisão do movimento). Na dismetria sacádica, o sujeito inicia o movimento normalmente, porém perde o alvo durante sua execução, sendo necessária a realização de sacadas corretivas para reposicionar a fóvea no alvo. A dismetria sacádica pode ser dividida em hipométrica - quando o movimento sacádico termina 
Movimentos Oculares no Bebê: o que Eles nos Indicam Sobre o Status Oftalmológico...

aquém do alvo - e hipermétrica - quando o movimento termina além do alvo (Harris et al., 1994).

Hipometria sacádica é freqüentemente encontrada em doenças cerebelares, principalmente na ataxia espinocerebelar dominante do tipo 3 e nos casos de ataxia oculomotora. Nos bebês, a persistência de hipometria após os 7 meses de idade é muito sugestiva de doença neurológica (Botzel, Rottach, $\&$ Buttner, 1993). Nos casos de perda de hemicampo visual, podem ocorrem múltiplas sacadas hipométricas apenas unilaterais (para o lado do campo visual sem visão), e, no caso de doenças do gânglio basal, as sacadas voluntárias geralmente são hipométricas.

As sacadas hipermétricas são muito menos comuns e estão, geralmente, associadas a doenças cerebelares como a ataxia espinocerebelar dominante do tipo 1 e lesões do tronco encefálico (Botzel et al., 1993; Rivaud-Pechoux et al., 1998).

Sacadas lentificadas são difíceis de diagnosticar, mas indicam o envolvimento da FRPP e, muito raramente, lesões em colículo superior (Hikosaka, 1989). Essa situação também pode ser encontrada em doenças mitocondriais, miastenia gravis e ataxia espinocerebelar dominante do tipo 2 (Adams, Starkman, \& Pulst, 1997; Katsanis, Iacomo, \& Harris, 1998). Em alguns casos, as sacadas horizontais estão preservadas, mas as sacadas verticais apresentam uma lentificação, como ocorre na doença de Gaucher tipo 3 e em alguns casos de distrofia muscular de Becker (Scelsa, Simpson, Reichler, \& Dai, 1996).

Dificuldade em iniciar uma sacada é, de todos os sinais acima descritos, o mais importante, porque está associado com muitas síndromes e desordens neurológicas (Tabela 1). Essa falha pode ser congênita ou adquirida e, muito freqüentemente, afeta somente as sacadas horizontais (Shawkat et al., 1995). Por ser um sinal clínico extremamente importante, a evolução de doenças neurodegenerativas pode ser monitorada pela sua intermitência, culminando numa constante incapacidade de gerar movimentos sacádicos nos estágios mais avançados (Regal, 1983). 


\section{Marcelo Fernandes Costa}

Tabela 1: Desordens neurológicas associadas à falha em iniciar sacadas.

\section{Problemas Perinatais}

Paralisia cerebral

Hipoxia

Hidrocefalia

Malformações Congênitas

Agenesia de corpo caloso

Macrocerebelo

Dilatação do quarto ventrículo

Hipoplasia de vermis cerebelar

Microcefalia

Encefalocele

Doenças Neurodegenerativas

Doenças de Refsum

Leucodistrofia de Krabbe

Ataxia telangectasia

Doença de Huntington

Doenças de Wilson

Doenças Adquiridas

Encefalite herpética

Tumores de fossa posterior

Neurofibromatose tipo 1

\section{Movimento de perseguição lenta}

Alterações nos movimentos de perseguição lenta não ocorrem isoladamente. Lesões no córtex e no cerebelo afetam esse tipo de movimento, e tem efeito semelhante no nistagmo optocinético (Leigh \& Zee, 1991). Em crianças com maturação visual atrasada, não se observa a presença de movimento de perseguição lenta; no entanto, tais crianças apresentam resposta normal ao nistagmo optocinético. Interessante notar que, nessas crianças, a acuidade visual medida pelos potenciais visuais evocados é normal, indicando um atraso no desenvolvimento da função cortical extraestriatal (Ohtsuka, Igarashi, Maekawa, \& Nakagawa, 1991). 
Movimentos Oculares no Bebê: o que Eles nos Indicam Sobre o Status Oftalmológico...

Em crianças com estrabismo congênito, é comum observar uma persistência da assimetria naso-temporal. Essa assimetria também é encontrada em bebês com lesão em áreas corticais posteriores, córtex frontal, tálamo e cerebelo. Nesses casos, ocorre uma ausência de perseguição lenta para o lado da lesão. Sujeitos com lesão cerebelar, de ganglio basal e de grandes extensões corticais podem apresentar um movimento de perseguição lenta realizado por sacadas de pequena amplitude (Pierrot-Deseilligny, Rivaud, Samson, \& Cambon, 1989).

\section{Movimentos vergenciais}

Importante mencionar que as alterações vergenciais quase sempre ocorrem na infância e, na grande maioria das vezes, em associação com o estrabismo. Existem seis tipos de alterações vergenciais, sendo que quatro se relacionam aos movimentos convergentes (Archer et al., 1989).

O primeiro que mencionaremos é o espasmo de convergência. Essa alteração é caracterizada por momentos em que ocorre máxima convergência dos olhos, além de uma acomodação e miose pupilar constantes (Lennerstrand et al., 1982). Esses episódios podem durar de segundos a minutos. Tal situação pode ser induzida por uma miopia moderada ou alta ou por um estrabismo convergente de tamanho variável (Archer et al., 1989). Em adultos, os sintomas referidos são visão dupla (diplopia) e dor ocular aguda e intensa. Pode-se observar a existência de um nistagmo na tentativa de reposicionar o olho. Nesse caso, a presença desse tipo de alteração ocular está freqüentemente ligada a alterações psicológicas e/ou psiquiátricas.

Em bebês, esse tipo de alteração tem sido relacionado com a presença de alterações neurológicas como malformação de Arnold-Chiari, adenoma de pituitária, miastenia gravis, toxicidade de drogas anticonvulsivantes e lesões mesencefálicas, principalmente as decorrentes de traumatismos cranianos (Moster \& Hoening, 1989).

Muito freqüente na clínica oftalmológica é a presença de sujeitos com insuficiência de convergência. Em tal doença, observa-se uma fraca ação da convergência, reduzida amplitude de fusão para perto e, em alguns casos, um estrabismo divergente intermitente. Borramento visual para perto, cefaléia e 
dor ocular são os sintomas mais freqüentes (Kohel, Kristan, Simon, \& Barrows, 1986; Lennerstrand et al., 1982).

Em adultos, essa doença é encontrada em mais de $70 \%$ das pessoas que trabalham diariamente, por várias horas, utilizando a visão para perto (atividades que exijam muita leitura ou o uso constante de computadores). Nos bebês, esses quadros podem ocorrer após doenças sistêmicas persistentes, infecções de sistema nervoso central e trauma (Lennerstrand et al., 1982). Uma condição similar, porém muito mais severa, é a paralisia de convergência. Nos bebês, ela é altamente sugestiva de lesão intracraniana severa, como encefalite, neurossífilis, intoxicação ou desmielinização (Kohel et al., 1986).

Paralisia ou insuficiência de divergência indicam geralmente uma alteração neurológica como, por exemplo, tumores e aumento de pressão intracraniana por trauma ou hidrocefalia (Moore, Harbison, \& Stockbridge, 1975).

Alguns bebês, na tentativa de olhar para cima, apresentam uma grande convergência, retração do globo ocular para o interior da órbita craniana e um intenso nistagmo. Esta é uma situação muito importante de se mencionar, pois está relacionada com lesões mesencefálicas, em comissura posterior e, em particular, nos tumores de glândula pineal. Essa alteração vergencial também é encontrada em bebês com hidrocefalia que apresentam uma pressão intracraniana muito elevada (Ochs, Strak, Hoyt, \& D’Amico, 1979).

\section{Reflexo vestíbulo-ocular}

A ausência do reflexo vestíbulo-ocular é um forte indicativo de defeito visual sensorial. Bebês com a síndrome de Usher, uma associação entre cegueira, surdez e atraso do desenvolvimento, freqüentemente apresentam alterações nesse tipo de movimento ocular (Baloh, Yee, Kimm, \& Horumbia, 1981).

No entanto, devemos lembrar que esse movimento pode estar ausente no bebê saudável até por volta da terceira semana de vida. Se a ausência persistir, é um forte indicativo de maturação visual atrasada (Hoyt, Jastrzebsky, \& Marg, 1985; Lennerstrand et al., 1982). Meningites, alterações vestibulares e cerebelares também podem levar a alterações no reflexo vestíbulo-ocular (Baloh et al., 1981). 
Movimentos Oculares no Bebê: o que Eles nos Indicam Sobre o Status Oftalmológico...

\section{Nistagmo optocinético}

Uma pobre acuidade visual, seja ela causada por alterações refracionais da óptica ocular (Baloh, Yee, \& Horumbia, 1980) ou por alterações neurológicas como maturação visual atrasada ou nistagmo, influencia na redução desse movimento ocular (Lennerstrand et al., 1982).

Displasias corticais e lesões cerebelares podem contribuir para levar a alterações nos movimentos optocinéticos (Harris, Walker, \& Shawkat, 1993). Assimetria do nistagmo optocinético tem sido descrita em crianças de 4-5 meses de idade com lesões parietais (Jacobs, Shawkat, \& Harris, 1993).

\section{Considerações finais}

Anormalidades de movimentos oculares raramente ocorrem como fatos isolados, exceto nos casos em que a etiologia decorre de alterações refracionais da óptica ocular. Sendo assim, é extremamente importante estar atento aos movimentos oculares de nossas crianças, pois, como vimos, eles podem nos indicar a existência de uma alteração neurológica.

Como demonstramos, todos os tipos de movimentos oculares podem e devem ser avaliados em neonatos e bebês, já que tal avaliação é imprescindível para se obter um prognóstico visual. A detecção de alterações oculomotoras nos primeiros meses de vida permite aos profissionais elaborar estratégias de tratamento a fim de impedir ou minimizar problemas visuais conseqüentes de e secundários a tais alterações, visto que nos primeiros anos de vida ocorre um acelerado desenvolvimento do sistema visual e até mesmo curtos períodos de estimulação inapropriada podem acarretar graves prejuízos funcionais.

O impacto dessas alterações assume, muitas vezes, uma magnitude que extrapola as limitações visuais. É muito comum, na clínica oftalmológica e ortóptica, atendermos pacientes em início de idade escolar apresentando importantes problemas escolares (dificuldades em enxergar no quadro negro, escrita lentificada, problemas de leitura etc.) e psicológicos (comportamentais, emocionais etc.) que, em muitos casos, decorrem de alterações visuais e oculares ocorridas no início da infância e que não foram devidamente diagnosticadas. 


\title{
Marcelo Fernandes Costa
}

Por fim, enfatizamos a importância de se realizar um completo exame da motilidade ocular. Sutis movimentos oculares anormais não serão detectados a menos que sejam investigados, e tais anormalidades podem ter um diagnóstico significante e um significante prognóstico para a criança.

Costa, M. F. (2007). Infant ocular movements: What they show us about the ophthalmological and neurological status. Psicologia USP, 18(2), 47-61.

\begin{abstract}
Abnormal eye movements may be a result of early abnormal development or a sign of underlying neurological or neuromuscular disease. However, it is important to the clinician to know such abnormalities and to distinguish them from normal but immature eye movements. This paper intends to shed some light on the different types of ocular movements, on how to interpret them in healthy infants and what alterations are found in different visual and neurological diseases.
\end{abstract}

Index terms: Eye movements. Infants. Vision disorders.

Costa, M. F. (2007). Les mouvements oculaires petits : qu'ils nous montrent-ils du du statut ophtalmologique et neurologique. Psicologia USP, 18(2), 47-61.

Résumé: Les mouvements anormaux des yeux peuvent être entendus comme résultants d'un développement anormal tout court ou comme un signe de maladie neurologique ou neuromusculaire. Ainsi, il est très important que les praticiens puissent distinguer telles anomalies des mouvements des yeux normaux mais pas encore mûrs. Cet article a pour but de clarifier certaines différences entre les mouvements oculaires, en abordant des méthodes de détection chez les bébés sains et aussi les changements caracteristiques des maladies visuelles et neurologiques.

Most-clés: Mouvement oculaire. Bébé. Troubles de la vision. 
Movimentos Oculares no Bebê: o que Eles nos Indicam Sobre o Status Oftalmológico...

\section{Referências}

Adams, C., Starkman, S., \& Pulst, S. M. (1997). Clinical and molecular analysis of a pedigree of southern Italian ancestry with spinocerebellar ataxia type 2. Neurology, 49, 1163 1166.

Archer, S. M., Sondhi, N., \& Helveston, E. M. (1989). Strabismus in infancy. Ophthalmology, 86, 133-137.

Atkinson, J. (1989). Development of the optokinetic nystagmus in the human infant and monkey infant: An analogue to development in kittens. Developmental Neurology, 79, 227-257.

Baloh, R. W., Yee, R. D., \& Horumbia, V. (1980). Optokinetic asymmetry in patients with maldevelopment foveas. Brain Research, 186, 211-216.

Baloh, R. W., Yee, R. D., Kimm, J., \& Horumbia, V. (1981). Vestibular-ocular reflex in patients with lesions involving the vestibulocerebellum. Experimental Neurology, 72, 141-152.

Barnes, G. R. (1983). Visual-vestibular interaction in the control of head and eye movement: The role of the visual feedback and predictive mechanisms. Progress in Neurobiology, $41,435-472$.

Botzel, K., Rottach, K., \& Buttner, U. (1993). Normal and pathological saccadic dysmetria. Brain, 116, 337-353.

Braddick, O. J., \& Atkinson, J. (1983). Some recent findings on the development of human binocularity: A review. BehaviouralBrain Research, 10, 141-150.

Carpenter, R. H. S. (1991). (Eds.). The neurology of eyes movement. Philadelphia: FA Davis.

Cordero, L., Clark, D. L., \& Urrutia, J. G. (1983). Postrotatory niystagmus in the full term and premature infant. International Journal of Pediatric Otorhinolaryngology, 5, 47-57.

Fries, W. (1984). Cortical projections to the superior colliculus in the macaque monkey: A retrograde study using horseradish peroxidase. Journal of Comparative Neurology, 230, 55-76.

Harris, C., Jacobs, M, \& Taylor, D. (1994). The development of bi-ocular and monocular optokinetic gain from 1-7 months. Investigative Ophthalmology and Visual Science, 35(Suppl.), 1829.

Harris, C., Jacobs, M, Shawkat, F., \& Taylor, D. (1993). The development of the saccadic accuracy in the first seven months. Clinical Visual Science, 8, 85-96.

Harris, C., Taylor, D., \& Vellodi, A. (1997). Eye movement recording in type 3 Gaucher disease: A preliminary report. Gaucher Clinical Perspectives, 5, 7-10. 


\section{Marcelo Fernandes Costa}

Harris, C., Walker, J., \& Shawkat, F. (1993). Eye movement in vestibulocerebellar disorder. Neuropedriatrics, 24, 117-122.

Hikosaka, O. (1989). Role of the basal ganglia in saccades. Review Neurology, 145, 580586.

Hoyt, C. S., Jastrzebsky, G., \& Marg, E. (1985). Delayed visual maturation in infancy. British Journal of Ophthalmology, 67, 127-130.

Jacobs, M., Shawkat, F., \& Harris, C. (1993). Eye movement and electrophysiological findings in infant with hemispheric pathology. Developmental Medicine and Child Neurology, 35, 431-435.

Katsanis, J., Iacomo, W. G., \& Harris, M. (1998). Development of oculomotor function in preadolescence, adolescence and adulthood. Psychophysiology, 35, 64-72.

Koh, P. S., Raffensperger, J. G., Berry, S., Larsen, M. B., Johnstone, H. S., Chou, P. et al. (1994). Long-term outcome in children with opsoclonus-myoclonus and ayaxia and coincident neuroblastoma. Journal of Pediatric, 125, 712-716.

Krohel, G. B., Kristan, R. W., Simon, J. W., \& Barrows, N. A. (1986). Posttraumatic convergence insufficiency. Annals of Ophthalmology, 18, 101-104.

Leigh, R. J., \& Zee, D. S. (Eds.). (1991). The neurology of eyes movement. Philadelphia: Davis.

Lennerstrand, G., Zee, D. S., \& Keller, E. L. (Eds.). (1982). Functional basis of ocular motility disorders. New York: Pergamon.

Moore, S., Harbison, J. W., \& Stockbridge, L. (1975). Divergence insufficiency. American Orthoptic Journal, 21, 59-63.

Morrow, M. J., \& Shape, J. A. (1990). Cerebral hemispheric localization of smooth pursuit asymmetry. Neurology, 40, 284-292.

Moschovakis, A. K., Scudder, C. A., \& Highstein, S. M. (1996). The miscroscopic anatomy and physiology of the mammalian saccadic system. Neurobiology, 50, 133-254.

Moster, M. L., \& Hoening, E. M. (1989). Spasm of the near reflex associated with metabolic encephalopathy. Neurology, 39, 150-159.

Ochs, A. L., Strak, L., Hoyt, W. F., \& D’Amico, D. (1979). Opposed adducting saccades in convergence-retraction nystagmus. Brain, 102, 497-508.

Ohtsuka, K., Igarashi, Y., Maekawa, H., \& Nakagawa, T. (1991). Pursuit deficit in bilateral pulvinar lesions. Ophthalmologica, 203, 169-202.

Pierrot-Deseilligny, C., Rivaud, S., Samson, Y., \& Cambon, H. (1989). Some cases involving the circuity of smooth ocular pursuit in the brainstem. Neuro-ophthalmology, 9, 31-42. 
Movimentos Oculares no Bebê: o que Eles nos Indicam Sobre o Status Oftalmológico...

Regal, D. M. (1983). The coordination of eye and hand movements during early infancy: A selective review. Behavioural Brain Research, 10, 125-132.

Rivaud-Pechoux, S., Durr, A., \& Gaymard, B. (1998). Eye movement abnormalities correlate with genotype in autossomal dominat cerebellar ataxia type I. Annals of Neurology, 43, 297-302.

Scelsa, S. N., Simpson, D. M., Reichler, B. D., \& Dai, M. (1996). Extraocular muscle invovement in Becker muscular dystrophy. Neurology, 46, 564-566.

Shawkat, F. S., Kingsley, D., Kendall, B., Russell Eggitt, I., Taylor, D. S. I., \& Harris, C. M. (1995). Neuroradiological and eye movements correlates in children with intermittent saccade failure: Ocular motor apraxia. Neuropediatrics, 24, 218-233.

Smith, J. L. (1987). Accomodative spasm versus spasm of the near reflex. Journal of Clinical Neuro-Ophthalmology, 7, 132-134.

Sokol, S., Peli, E., Moskowitz, A., \& Reese, D. (1991). Pursuit eye movement in late-onset esotropia. Journal of Pediatric Ophthalmology and Strabismus, 28, 82-86.

von Hofsten, C., \& Rosander, K. (1997). Development of the smooth pursuit tracking in young infants. Vision Research, 37, 1799-1810.

Recebido em: 9.05.2006

Aceito em: 7.08 .2006 
